\title{
A MATHEMATICAL MODEL AND NUMERICAL SOLUTION OF INTERFACE PROBLEMS FOR STEADY STATE HEAT CONDUCTION
}

\author{
Z. MURADOGLU SEYIDMAMEDOV AND EBRU OZBILGE
}

Received 28 March 2006; Revised 1 July 2006; Accepted 16 July 2006

We study interface (or transmission) problems arising in the steady state heat conduction for layered medium. These problems are related to the elliptic equation of the form $A u:=-\nabla(k(x) \nabla u(x))=F(x), x \in \Omega \subset \mathbb{R}^{2}$, with discontinuous coefficient $k=k(x)$. We analyse two types of jump (or contact) conditions across the interfaces $\Gamma_{\delta}^{-}=\Omega_{1} \cap \Omega_{\delta}$ and $\Gamma_{\delta}^{+}=\Omega_{\delta} \cap \Omega_{2}$ of the layered medium $\Omega:=\Omega_{1} \cup \Omega_{\delta} \cup \Omega_{2}$. An asymptotic analysis of the interface problem is derived for the case when the thickness $(2 \delta>0)$ of the layer (isolation) $\Omega_{\delta}$ tends to zero. For each case, the local truncation errors of the used conservative finite difference scheme are estimated on the nonuniform grid. A fast direct solver has been applied for the interface problems with piecewise constant but discontinuous coefficient $k=k(x)$. The presented numerical results illustrate high accuracy and show applicability of the given approach.

Copyright (c) 2006 Z. M. Seyidmamedov and E. Ozbilge. This is an open access article distributed under the Creative Commons Attribution License, which permits unrestricted use, distribution, and reproduction in any medium, provided the original work is properly cited.

\section{Introduction}

Interface problems arise in the setting of various physical and engineering problems (see [1-3, 7-9]) and references therein. Mathematical modelling of such steady state problems leads to the following elliptic problem with discontinuous coefficient $k(x)=$ $\left(k_{1}(x), k_{2}(x)\right)$ :

$$
\begin{gathered}
A u:=-\nabla(k(x) \nabla u)=F(x), \quad x=\left(x_{1}, x_{2}\right) \in \Omega \subset \mathbb{R}^{2}, \\
u(x)=0, \quad x \in \partial \Omega,
\end{gathered}
$$

where $\Omega:=\left\{\left(x_{1}, x_{2}\right) \in \mathbb{R}^{2}: x_{1} \in\left(-l_{1}, l_{1}\right), x_{2} \in\left(0, l_{2}\right), l_{i}>0\right\}$. The discontinuity of the 
coefficient $k=k(x)$ necessitates the consideration of the weak solution $u \in \stackrel{0}{H}^{1} \in(\Omega):=$ $\left\{u \in H^{1}(\Omega): u(x)=0, x \in \partial \Omega\right\}$ of problem (1.1), which satisfies the following integral identity [6]:

$$
\int_{\Omega} k(x) \nabla u \nabla v d x=\int_{\Omega} F(x) v(x) d x, \quad \forall v \in \stackrel{0}{H}^{1}(\Omega)
$$

Here $H^{1}(\Omega)$ is the Sobolev space [6]. The existence and uniqueness of the weak solution $u \in H^{1}(\Omega)$ of the Dirichlet problem (1.1) are outlined, for example, in [6]. In the classical formulation of problem (1.1), the solution $u(x)$ satisfies the elliptic equation (1.1) in $\Omega$, Dirichlet condition (1.1) on $\partial \Omega$, and the following transmission conditions "(ideal contact)" on the interface $\Gamma_{\xi}:=\left\{x_{1}=\xi\right\} \subset \Omega$ :

$$
\begin{gathered}
{[u]_{x_{1}=\xi}:=u\left(\xi+0, x_{2}\right)-u\left(\xi-0, x_{2}\right)=0, \quad \xi \in\left(-l_{1}, l_{1}\right),} \\
{\left[k_{1} \frac{\partial u}{\partial x_{1}}\right]_{x_{1}=\xi}:=\left(k_{1}^{(2)} \frac{\partial u}{\partial x_{1}}\right)\left(\xi+0, x_{2}\right)-\left(k_{1}^{(1)} \frac{\partial u}{\partial x_{1}}\right)\left(\xi-0, x_{2}\right)=0 .}
\end{gathered}
$$

The above conditions mean continuity of the temperature and the flux across the interface $\Gamma_{\xi}:=\left\{x_{1}=\xi\right\}$. Throughout this paper, the superscripts indicate the two limits from opposite sides of the interface $\Gamma_{\xi}$. Note that the transmission conditions (1.3) are not possible unique ones, that arise in practice. Most of the other physically possible situations for the elliptic problem (1.1) correspond to the following transmission conditions $[9-11,13]$ :

$$
\begin{gathered}
{[u]_{x_{1}=\xi}=0, \quad\left[k_{1} \frac{\partial u}{\partial x_{1}}\right]_{x_{1}=\xi}=-\alpha(x),} \\
\sigma[u]_{x_{1}=\xi}=\left(k_{1} \frac{\partial u}{\partial x_{1}}\right)\left(\xi, x_{2}\right), \quad\left[k_{1} \frac{\partial u}{\partial x_{1}}\right]_{x_{1}=\xi}=0 .
\end{gathered}
$$

In the first case, the flux is discontinuous at the contact surface (interface $\Gamma_{\xi}$ ), although the potential (or temperature) is continuous. The function $\alpha=\alpha(x)$ here corresponds to the distributed sources on the interface $\Gamma_{\xi}$. In the case of conditions (1.5), the flux is continuous, whereas the function $u=u(x)$ has discontinuity across the contact line $x_{1}=\xi$.

Such problems arise frequently in physical and engineering applications, in particular, in steady state heat conduction, diffusion or electrostatic problems in layered nonhomogeneous medium [13]. The main distinguished feature of these problems is the discontinuity of the heat conduction coefficient $k_{1}(x)$ in the elliptic equation (1.1).

The second-order homogeneous finite difference schemes for one-dimensional problems with discontinuous coefficients on uniform meshes using the jump conditions have been given first in [12]. Later, various immersed methods have been developed for numerical solution of such interface problems (see $[5,9-11,14]$ and references therein). 
In one of the previous studies (see [4]), an immersed finite element space is used to solve the elliptic interface problems by a finite volume element method. Special nodal basis functions are introduced in a triangle whose interior intersects with the interface so that the jump conditions are satisfied. Further, finite difference methods for elliptic equations of the form $\nabla(\beta(x) \nabla u)+\kappa(x) u(x)=f(x)$ in a region $\Omega$ in 1 or 2 space dimensions are developed in [9]. Here $\Omega$ is assumed to be a simple region (e.g., a rectangle) and a uniform rectangular grid is used. Across the irregular surface $\Gamma$ of codimension 1 contained in $\Omega$ the functions $\beta$, $\kappa$, and $f$ are assumed to be discontinuous, and along $\Gamma$, the source $f$ may have a delta function. An immersed interface elliptic problem with discontinuities and singularities in a circular domain has been studied in [10]. On the contact surface, the flux and the potential (or temperature) here are taken discontinuous.

We consider more general problem where the domain consists of the following three parts: conductor-isolator-conductor (see, Figure 2.1(b)). Evidently, the interface problem is a limit case of this problem. We provide an asymptotic analysis of the problem conductor-isolator-conductor and show which interface problem is a limit case. Then we consider an interface problem with ideal contact conditions. Since thickness of the isolation is small enough with respect to the dimension of the domain isolation, our approach is based on conservative finite difference schemes not on uniform, but on a nonuniform, mesh with the same order of accuracy.

In this paper, we consider the following three types of interface problems:

(P1) the interface problem for two-layered nonhomegeneous medium with ideal contact conditions (1.3),

(P2) the interface problem for nonhomegeneous three-layered medium (conductorisolation-conductor) with ideal contact conditions (1.3),

(P3) the interface problem for two-layered nonhomegeneous medium with continuous flux and discontinuous temperature across the interface.

In the first part of the paper, we prove that the problem (P3) is a limit case of the problem (P2), when the thickness of the isolation tends to zero. In the second part, we derive the conservative finite difference schemes of a nonuniform mesh, which has a truncation error of order $O\left(h^{2}\right)$. Then we obtain the finite difference schemes of orders $O(h)$ and $O\left(h^{2}\right)$ on the interface for Neumann transmission conditions. In the final part, we illustrate numerical results related to considered problems.

\section{The mathematical model of layered conductivities without isolation: ideal contact}

Let us consider the steady state heat conduction problem

$$
\begin{gathered}
-\frac{\partial}{\partial x_{1}}\left(k_{1}(x) \frac{\partial u}{\partial x_{1}}\right)-\frac{\partial}{\partial x_{2}}\left(k_{2}(x) \frac{\partial u}{\partial x_{2}}\right)=F(x), \quad x \in \Omega_{i}, \\
{[u]_{x_{1}=\xi}=0, \quad\left[k_{1} \frac{\partial u}{\partial x_{1}}\right]_{x_{1}=\xi}=0, \quad x \in \Gamma_{\xi},} \\
u(x)=0, \quad x \in \partial \Omega,
\end{gathered}
$$




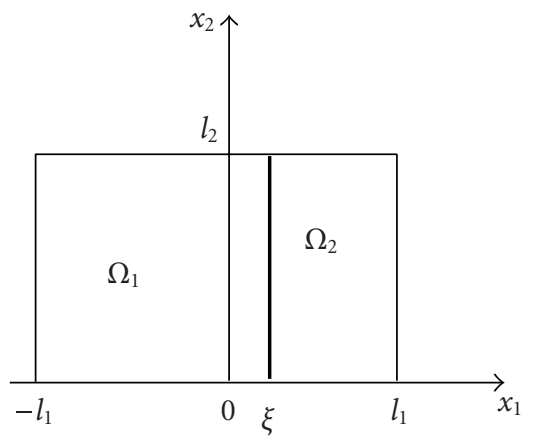

(a)

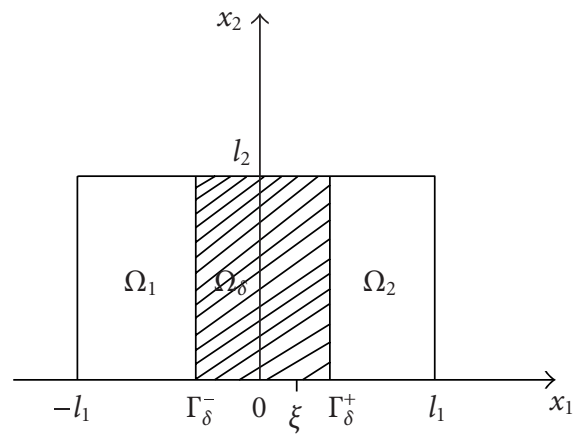

(b)

Figure 2.1. The geometry of layered conductivities without isolation and with thin isolation is given in part (a) and part (b), respectively.

in the domain $\Omega=\Omega_{1} \cup \Gamma_{\xi} \cup \Omega_{2}$, occupied by the homegeneous bodies $\Omega_{1}$ and $\Omega_{2}$, with different heat conductivities

$$
k_{1}(x)= \begin{cases}k_{1}^{(1)}, & x \in \Omega_{1}, \\ k_{1}^{(2)}, & x \in \Omega_{2}, k_{1}^{(i)}=\mathrm{const}\end{cases}
$$

along the $O x_{1}$-axis and $k_{2}(x)$ along the $O x_{2}$-axis. The piecewise constant coefficient $k_{1}(x)$ is assumed to be discontinuous $\left(k_{1}^{(1)} \neq k_{1}^{(2)}\right)$ and the coefficient $k_{2}(x)>0$ is assumed to be continuously differentiable function. The source function $F(x)$ is assumed to be continuous. The contact conditions between the bodies $\Omega_{1}$ and $\Omega_{2}$ are assumed to be ideal ones via the interface

$$
\Gamma_{\xi}:=\left\{\left(\xi, x_{2}\right) \in \mathbb{R}^{2}: \xi \in\left(-l_{1}, l_{1}\right), x_{2} \in\left(0, l_{2}\right)\right\}
$$

Hence we assume that the layered body occupies the domain $\Omega$ with the boundary $\partial \Omega:=$ $\Gamma_{1} \cup \Gamma_{2} \cup \Gamma_{3} \cup \Gamma_{4}$ (Figure 2.1(a)), where $\Gamma_{1}=\left(-l_{1}, l_{1}\right) \times\left\{x_{2}=0\right\}, \Gamma_{2}=\left\{x_{1}=l_{1}\right\} \times\left(0, l_{2}\right)$, $\Gamma_{3}=\left(-l_{1}, l_{1}\right) \times\left\{x_{2}=l_{2}\right\}$, and $\Gamma_{4}=\left\{x_{1}=-l_{1}\right\} \times\left(0, l_{2}\right)$.

Then we can prove that the solution $u \in C^{2}\left(\Omega_{1} \cup \Omega_{2}\right) \cap C(\bar{\Omega})$ of the interface problem is also the solution of the variational problem (1.2) given by (2.4) for discontinuous coefficient $k_{1}(x)$.

Theorem 2.1. Let $u \in C^{2}\left(\Omega_{1} \cup \Omega_{2}\right) \cap C^{0}(\bar{\Omega})$ be the solution of the interface problem (2.1)(2.3). Then $u(x)$ is also the solution of the variational problem (1.2), for the discontinuous coefficient $k_{1}(x)$ given by (2.4). 
Proof. Let us multiply the both sides of (2.1) by $v=v(x)$, integrate them on $\Omega_{1}$ and $\Omega_{2}$ seperately, then apply integration by parts,

$$
\begin{aligned}
\int_{0}^{l_{2}} \int_{-l_{1}}^{\xi} k(x) \nabla u \nabla v d x_{1} d x_{2}= & \int_{0}^{l_{2}}\left(k_{1}^{(1)}(x) \frac{\partial u}{\partial x_{1}} v\right)_{x_{1}=-l_{1}+0}^{x_{1}=\xi-0} d x_{2}+\int_{-l_{1}}^{\xi}\left(k_{2}(x) \frac{\partial u}{\partial x_{2}} v\right)_{x_{2}=+0}^{x_{2}=l_{2}-0} d x_{1} \\
& +\int_{0}^{l_{2}} \int_{-l_{1}}^{\xi} F(x) v(x) d x_{1} d x_{2}, \\
\int_{0}^{l_{2}} \int_{\xi}^{l_{1}} k(x) \nabla u \nabla v d x_{1} d x_{2}= & \int_{0}^{l_{2}}\left(k_{1}^{(2)}(x) \frac{\partial u}{\partial x_{1}} v\right)_{x_{1}=\xi+0}^{x_{1}=l_{1}-0} d x_{2}+\int_{\xi}^{l_{1}}\left(k_{2}(x) \frac{\partial u}{\partial x_{2}} v\right)_{x_{2}=+0}^{x_{2}=l_{2}-0} d x_{1} \\
& +\int_{0}^{l_{2}} \int_{\xi}^{l_{1}} F(x) v(x) d x_{1} d x_{2} .
\end{aligned}
$$

Taking into account the homogeneous Dirichlet condition (2.3) and summing up the identities, we get

$$
\begin{aligned}
\int_{0}^{l_{2}} \int_{-l_{1}}^{l_{1}} k(x) \nabla u \nabla v d x_{1} d x_{2} \\
\quad=\int_{0}^{l_{2}}\left[\left(k_{1}^{(1)}(x) \frac{\partial u}{\partial x_{1}} v\right)_{x_{1}=\xi-0}-\left(k_{1}^{(2)}(x) \frac{\partial u}{\partial x_{1}} v\right)_{x_{1}=\xi+0}\right] d x_{2} \\
\quad+\int_{0}^{l_{2}} \int_{-l_{1}}^{l_{1}} F(x) v(x) d x_{1} d x_{2}
\end{aligned}
$$

for all $v=v(x)$. Since $u=u(x)$ satisfies the Dirichlet transmission condition $[u]_{x_{1}=\xi}=0$, it is natural to require that arbitrary function $v=v(x)$ also satisfies this condition. Using this condition in the first integral on the right-hand side, we obtain

$$
\int_{0}^{l_{2}} \int_{-l_{1}}^{l_{1}} k(x) \nabla u \nabla v d x_{1} d x_{2}=\int_{0}^{l_{2}}\left(k_{1}(x) \frac{\partial u}{\partial x_{1}}\right)_{x_{1}=\xi+0}^{x_{1}=\xi-0} v\left(\xi, x_{2}\right) d x_{2}+\int_{\Omega} F(x) v(x) d x .
$$

This integral idenditity with the Neumann transmission condition completes the proof.

This theorem shows the equivalence of problem (1.1), with the discontinuous coefficient $k_{1}(x)$ given by (2.4), and the transmission problem (2.1)-(2.3), although problem (1.1) does not contain any transmission condition. This suggests a possibility of construction of such a finite difference analogue of the interface problem, which has the similar structure. Specifically, the problem is to construct the homogeneous finite difference scheme, which has the same form for all mesh points, including ones on the interface $\Gamma_{\xi}$. 
6 Numerical analysis of steady state interface problems

\section{The interface problem for layered conductivities with isolation: asymptotic analysis}

Consider now the steady state heat conduction problem

$$
\begin{gathered}
-\frac{\partial}{\partial x_{1}}\left(k_{1}(x) \frac{\partial u}{\partial x_{1}}\right)-\frac{\partial}{\partial x_{2}}\left(k_{2}(x) \frac{\partial u}{\partial x_{2}}\right)=F(x), \quad x \in \Omega_{i}, \\
-\gamma\left(\frac{\partial^{2} u}{\partial x_{1}^{2}}+\frac{\partial^{2} u}{\partial x_{2}^{2}}\right)=F(x), \quad x \in \Omega_{\delta}, \\
{[u]_{x_{1}=-\delta}=0, \quad\left(\gamma \frac{\partial u}{\partial x_{1}}\right)_{x_{1}=-\delta+0}-\left(k_{1}^{(1)} \frac{\partial u}{\partial x_{1}}\right)_{x_{1}=-\delta-0}=0} \\
{[u]_{x_{1}=+\delta}=0, \quad\left(k_{1}^{(2)} \frac{\partial u}{\partial x_{1}}\right)_{x_{1}=+\delta+0}-\left(\gamma \frac{\partial u}{\partial x_{1}}\right)_{x_{1}=+\delta-0}=0} \\
u(x)=0, \quad x \in \partial \Omega
\end{gathered}
$$

in the domain $\Omega:=\Omega_{1} \cup \Omega_{\delta} \cup \Omega_{2}$, formed by the homogeneous conductive bodies occupying the domains

$$
\begin{gathered}
\Omega_{1}:=\left\{\left(x_{1}, x_{2}\right) \in \mathbb{R}^{2}: x_{1} \in\left(-l_{1},-\delta\right), x_{2} \in\left(0, l_{2}\right)\right\}, \\
\Omega_{2}:=\left\{\left(x_{1}, x_{2}\right) \in \mathbb{R}^{2}: x_{1} \in\left(+\delta, l_{1}\right), x_{2} \in\left(0, l_{2}\right)\right\}, \quad l_{i}>0, \delta>0,
\end{gathered}
$$

with conductivities $k_{1}^{(1)}(x)$ and consider $k_{1}^{(2)}(x)$, respectively, and the isolation between $\Omega_{1}$ and $\Omega_{2}$ occupying the domain (Figure 2.1(b)),

$$
\Omega_{\delta}:=\left\{\left(x_{1}, x_{2}\right) \in \mathbb{R}^{2}: x_{1} \in(-\delta, \delta), x_{2} \in\left(0, l_{2}\right)\right\} .
$$

It is assumed that the conductivity of the isolation, with the thickness $2 \delta>0$, is small enough, $\gamma=$ const $\ll 1$.

We assume that heat transfers between conductivities and the isolation across the interfaces

$$
\Gamma_{\delta}^{-}=\left\{\left(-\delta, x_{2}\right): x_{2} \in\left(0, l_{2}\right)\right\}, \quad \Gamma_{\delta}^{+}=\left\{\left(\delta, x_{2}\right): x_{2} \in\left(0, l_{2}\right)\right\}
$$

according to ideal contact conditions (3.3)-(3.4).

Introducing the piecewise continuous coefficient

$$
k_{1}(x)= \begin{cases}k_{1}^{(1)}(x), & x \in \Omega_{1}, \\ \gamma, & x \in \Omega_{\delta}, \\ k_{1}^{(2)}(x), & x \in \Omega_{2},\end{cases}
$$

we can show, as in the proof of Theorem 2.1, that the solution $u(x) \in C^{2}\left(\Omega_{1} \cup \Omega_{2}\right) \cap$ $C(\bar{\Omega})$ of problem (3.1)-(3.5) is also the solution of the variational problem (1.2), for given coefficient (3.9). 
In the case of the finite thickness isolation $\Omega_{\delta}$, problem (3.1)-(3.5) represents an interface problem with two ideal contact conditions across the interfaces $\Gamma_{\delta}^{-}$and $\Gamma_{\delta}^{+}$. In practice, the isolator can be given as a thin boundary layer with small enough thickness $\delta>0$. If the value of the thickness is less than the mesh size $h_{1}$ along the direction $O x_{1}$, that is, $2 \delta<h_{1}$, then the interface conditions (3.3)-(3.4) cannot be approximated on this mesh. To derive a finite difference approximation of the interface problem (3.1)-(3.5) for this case, one needs to derive an asymptotic analysis of this problem, when $\delta \rightarrow 0$.

Proposition 3.1. The limit case, when $\delta \rightarrow 0$, of the transmission problem (3.1)-(3.5) with ideal contact conditions, is the following transmission problem with nonideal contact conditions:

$$
\begin{gathered}
-\frac{\partial}{\partial x_{1}}\left(k_{1}(x) \frac{\partial u}{\partial x_{1}}\right)-\frac{\partial}{\partial x_{2}}\left(k_{2}(x) \frac{\partial u}{\partial x_{2}}\right)=F(x), \quad x \in \Omega_{i}, \\
\sigma[u]_{x_{1}=0}=\left(k_{1}^{(1)}(x) \frac{\partial u}{\partial x_{1}}\right)_{x_{1}=-0}, \quad\left(k_{1}^{(1)}(x) \frac{\partial u}{\partial x_{1}}\right)_{x_{1}=-0}=\left(k_{1}^{(2)}(x) \frac{\partial u}{\partial x_{1}}\right)_{x_{1}=+0}, \quad x \in \Gamma_{0}, \\
u(x)=0, \quad x \in \partial \Omega .
\end{gathered}
$$

Proof. Let us assume that $u=u(x)$ is the solution of the transmission problem (3.1)(3.5). Integrating (3.1) on $\Omega_{1}$, and (3.2) on $\Omega_{\delta}^{-}:=\left\{\left(x_{1}, x_{2}\right) \in \Omega_{\delta}: x_{1} \in(-\delta, \xi), \xi \in\right.$ $(-\delta, \delta)\}$, where $\xi \in(-\delta, \delta)$ and $\delta>0$ is an arbitrary small parameter and summing up the obtained integral identities imply

$$
\begin{aligned}
& \int_{0}^{l_{2}}\left\{\int_{-l_{1}}^{-\delta} \frac{\partial}{\partial x_{1}}\left(k_{1}^{(1)}(x) \frac{\partial u}{\partial x_{1}}\right) d x_{1}+\int_{-\delta}^{\xi} \gamma \frac{\partial^{2} u}{\partial x_{1}^{2}} d x_{1}\right\} d x_{2} \\
& \quad+\int_{0}^{l_{2}}\left\{\int_{-l_{1}}^{-\delta} \frac{\partial}{\partial x_{2}}\left(k_{2}(x) \frac{\partial u}{\partial x_{2}}\right) d x_{1}+\int_{-\delta}^{\xi} \gamma \frac{\partial^{2} u}{\partial x_{2}^{2}} d x_{1}\right\} d x_{2}+\int_{\Omega_{\delta}^{-} \cup \Omega_{1}} F(x) d x=0 .
\end{aligned}
$$

We apply here the mean value theorem, dividing first the both sides of the above integral identity by $l_{2} \neq 0$. Then we have

$$
\begin{aligned}
k_{1}^{(1)}( & \left.-\delta-0, \tilde{x}_{2}\right) \frac{\partial u}{\partial x_{1}}\left(-\delta-0, \tilde{x}_{2}\right)-k_{1}^{(1)}\left(-l_{1}+0, \tilde{x}_{2}\right) \frac{\partial u}{\partial x_{1}}\left(-l_{1}+0, \tilde{x}_{2}\right)+\gamma \frac{\partial u}{\partial x_{1}}\left(\xi-0, \tilde{x}_{2}\right) \\
& -\gamma \frac{\partial u}{\partial x_{1}}\left(-\delta+0, \tilde{x}_{2}\right)+\frac{1}{l_{2}} \int_{0}^{l_{2}}\left\{\int_{-l_{1}}^{-\delta} \frac{\partial}{\partial x_{2}}\left(k_{2}(x) \frac{\partial u}{\partial x_{2}}\right) d x_{1}+\int_{-\delta}^{\xi} \gamma \frac{\partial^{2} u}{\partial x_{2}^{2}} d x_{1}\right\} d x_{2} \\
& +\frac{1}{l_{2}} \int_{\Omega_{\delta}^{-} \cup \Omega_{1}} F(x) d x=0,
\end{aligned}
$$


8 Numerical analysis of steady state interface problems

where $\tilde{x}_{2} \in\left(0, l_{2}\right)$. We use here the second interface condition $(3.3)$ for the flux on $\Gamma_{\delta}^{-}$:

$$
\begin{aligned}
\gamma \frac{\partial u}{\partial x_{1}} & \left(\xi-0, \tilde{x}_{2}\right)-k_{1}^{(1)}\left(-l_{1}+0, \tilde{x}_{2}\right) \frac{\partial u}{\partial x_{1}}\left(-l_{1}+0, \tilde{x}_{2}\right) \\
& +\frac{1}{l_{2}} \int_{0}^{l_{2}}\left\{\int_{-l_{1}}^{-\delta} \frac{\partial}{\partial x_{2}}\left(k_{2}(x) \frac{\partial u}{\partial x_{2}}\right) d x_{1}+\int_{-\delta}^{\xi} \gamma \frac{\partial^{2} u}{\partial x_{2}^{2}} d x_{1}\right\} d x_{2}+\frac{1}{l_{2}} \int_{\Omega_{\delta}^{-} \cup \Omega_{1}} F(x) d x=0 .
\end{aligned}
$$

Going to the limit as $-l_{1} \rightarrow-\delta-0$ (the first integral drops), we obtain

$$
\begin{gathered}
\gamma \frac{\partial u}{\partial x_{1}}\left(\xi-0, \tilde{x}_{2}\right)-k_{1}^{(1)}\left(-\delta-0, \tilde{x}_{2}\right) \frac{\partial u}{\partial x_{1}} k_{1}^{(1)}\left(-\delta-0, \tilde{x}_{2}\right) \\
+\frac{1}{l_{2}} \int_{0}^{l_{2}}\left[\int_{-\delta}^{\xi}\left(\gamma \frac{\partial^{2} u}{\partial x_{2}^{2}}+F\left(x_{1}, x_{2}\right)\right) d x_{1}\right] d x_{2}=0 .
\end{gathered}
$$

Since the parameter $\xi \in(-\delta, \delta)$ is an arbitrary one, integrating the both sides of the above identity with respect to this parameter on $(-\delta, \delta)$, we obtain

$$
\begin{aligned}
\gamma[u( & \left.\left.+\delta, \tilde{x}_{2}\right)-u\left(-\delta, \tilde{x}_{2}\right)\right]-2 \delta k_{1}^{(1)}\left(-\delta-0, \tilde{x}_{2}\right) \frac{\partial u}{\partial x_{1}}\left(-\delta-0, x_{2}\right) \\
& +\int_{-\delta}^{\delta}\left\{\frac{1}{l_{2}} \int_{0}^{l_{2}} \int_{-\delta}^{\xi}\left(\gamma \frac{\partial^{2} u}{\partial x_{2}^{2}}+F\left(x_{1}, x_{2}\right)\right) d x_{1} d x_{2}\right\} d \xi=0 .
\end{aligned}
$$

Let us divide now the both sides by $2 \delta \neq 0$. Then going to the limit as $\delta, \gamma \rightarrow 0$ and requiring $\sigma:=\gamma /(2 \delta)=$ const, we get

$$
\sigma\left[u\left(+0, \tilde{x}_{2}\right)-u\left(-0, \tilde{x}_{2}\right)\right]=k_{1}^{(1)}\left(-0, \tilde{x}_{2}\right) \frac{\partial u}{\partial x_{1}}\left(-0, \tilde{x}_{2}\right), \quad \tilde{x}_{2} \in\left(0, l_{2}\right),
$$

where $\xi \rightarrow 0$ as $\delta \rightarrow 0$. This condition can be rewritten in the following form:

$$
\sigma[u]_{x_{1}=0}=\left(k_{1}^{(1)} \frac{\partial u}{\partial x_{1}}\right)_{x_{1}=-0} .
$$

Integrating (3.1) and (3.2) on $\Omega_{2}$ and $\Omega_{\delta}^{+}:=\left\{\left(x_{1}, x_{2}\right) \in \Omega_{\delta}: x_{1} \in(\xi, \delta)\right\}$, respectively, by the same way, we can obtain the second limit condition

$$
\sigma[u]_{x_{1}=0}=\left(k_{1}^{(2)} \frac{\partial u}{\partial x_{1}}\right)_{x_{1}=+0}
$$

Conditions (3.17)-(3.18) imply the transmission conditions (3.10). This completes the proof.

Conditions (3.17) and (3.18) imply that the flux

$$
\varphi_{0}\left(x_{2}\right):=\left[k_{1}(x) \frac{\partial u}{\partial x_{1}}\right]_{x_{1}=0}
$$


across the interface $\Gamma_{0}=\left\{\left(0, x_{2}\right) \in \mathbb{R}^{2}: x_{2} \in\left(0, l_{2}\right)\right\}$ remains continuous, when the thickness tends to zero. However, as these conditions show, the temperature $u(x)$ becomes discontinuous across the interface $\Gamma_{0}$. The jump of the function $u(x)$ across the interface is expressed via the flux $\varphi_{0}\left(x_{2}\right)$ on $\Gamma_{0}$ by formula (3.19).

The above asymptotic analysis shows that the limit case $\delta \rightarrow 0$ of the interface problem (3.1)-(3.5) with ideal contact conditions (3.3)-(3.4) is the interface problem (3.10) whose solution has discontinuity across the interface. In practice, this analysis is necessary, especially for the class of problems, in which the conductivity $\gamma>0$ and the thickness $2 \delta>0$ are of the same order of small parameters.

\section{Conservative finite difference schemes for interface problems on nonuniform mesh}

Our goal here is a finite difference approximation of transmission problems (2.1)-(2.3) and (3.10). For simplicity, we assume $\xi=0$, so the interface $\Gamma_{\xi}$ of the domain $\Omega$, shown in Figure 2.1(a), lies on $\mathrm{O} x_{2}$-axis. The most existing numerical approaches use a uniform mesh and the interface points $\left(\xi, x_{2}\right), x_{2} \in\left(0, l_{2}\right)$ are assumed to be between mesh points $x_{i_{0}}-j<\xi<x_{i_{0}}+j$ (see, e.g., [9]). We will develop here finite difference equations on a nonuniform mesh, assuming that the interface lies on mesh points.

Let us denote by

$$
\widehat{w}_{h}:=\left\{\left(x_{1}^{\left(i_{1}\right)}, x_{2}^{\left(i_{2}\right)}\right) \in \Omega: x_{1}^{\left(i_{1}\right)}=-l_{1}+i_{1} h_{1}^{\left(i_{1}\right)}, x_{2}^{\left(i_{2}\right)}=i_{2} h_{2}^{\left(i_{2}\right)}, i_{1}=\overline{0, N_{1}+1}, i_{2}=\overline{0, N_{2}+1}\right\}
$$

a nonuniform rectangular mesh with mesh steps $h_{j}^{\left(i_{p}\right)}>0, j=1,2$, assuming that $x_{1}^{(0)}=$ $-l_{1}, x_{1}^{\left(N_{1}+1\right)}=l_{1}, x_{2}^{(0)}=0, x_{2}^{\left(N_{2}+1\right)}=l_{2}$. Then, according to the above assumption, the interface mesh points are defined as follows: $\left(0, x_{2}^{\left(i_{2}\right)}\right):\left(x_{1}^{\left(i_{1}\right)}, x_{2}^{\left(i_{2}\right)}\right) \in \Gamma_{\xi}, i_{2}=\overline{1, N_{2}}$.

Below, we will use the following notations without index at the mesh point $\left(x_{1}^{\left(i_{1}\right)}, x_{2}^{\left(i_{2}\right)}\right)$ :

$$
h_{p}:=h_{p}^{\left(i_{p}\right)}=x_{p}^{\left(i_{p}\right)}-x_{p}^{\left(i_{p}-1\right)}, \quad h_{p}^{ \pm}:=h_{p}^{\left(i_{p} \pm 1\right)}, \quad \hbar_{p}=0.5\left(h_{p}^{-}+h_{p}^{+}\right), \quad p=1,2 .
$$

For the first left and right finite differences, we will use the standard notations

$$
\begin{aligned}
& u_{x_{1}}:=\frac{u^{\left(+1_{1}\right)}-u}{h_{1}^{+}} \equiv \frac{u\left(x_{1}+h_{1}^{+}, x_{2}\right)-u\left(x_{1}, x_{2}\right)}{h_{1}^{+}}, \\
& u_{\bar{x}_{1}}:=\frac{u-u^{\left(-1_{1}\right)}}{h_{1}^{-}} \equiv \frac{u\left(x_{1}, x_{2}\right)-u\left(x_{1}-h_{1}^{-}, x_{2}\right)}{h_{1}^{-}} .
\end{aligned}
$$

In a similar way, $u_{x_{2}}$ and $u_{\bar{x}_{2}}$ can also be defined. The first finite difference corresponding to the mesh step $\hbar_{p}=0.5\left(h_{p}^{-}+h_{p}^{+}\right)$will be denoted as follows: $u_{\widehat{x}_{p}}:=\left(u^{\left(+1_{p}\right)}-u\right) / \hbar_{p}, p=$ 1,2 .

We define the mesh point $\left(x_{1}^{\left(i_{1}\right)}, x_{2}^{\left(i_{2}\right)}\right) \in \widehat{w}_{h}$ to be a regular mesh point, if the interface $\Gamma_{\xi}$ does not come between any points in the standard five-point stencil (Figure 4.1). In view of these definitions, the standard five-point conservative finite difference scheme approximating the elliptic operator $(1.1)$ at the regular mesh point $\left(x_{1}^{\left(i_{1}\right)}, x_{2}^{\left(i_{2}\right)}\right)$, with the 


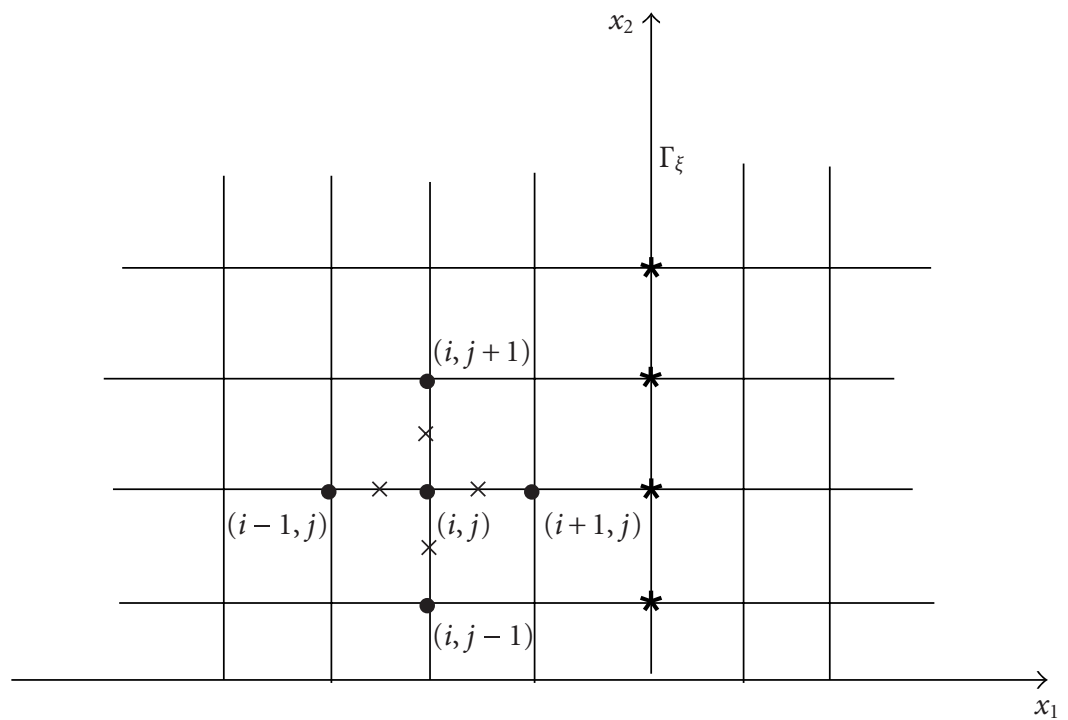

Figure 4.1. Standard five-point stencil near the interface $\Gamma_{\xi}$.

coefficient $k_{1}(x), x \in \Omega$, is defined as follows $[9,11]$ :

$$
A_{h} y:=-\left(a_{1} y_{\bar{x}_{1}}\right)_{\widehat{x}_{1}}-\left(a_{2} y_{\bar{x}_{2}}\right)_{\widehat{x}_{2}} \text {. }
$$

Here the coefficients $a_{p}$ are defined, for example, by the formulas

$$
a_{1}(x)=k_{1}\left(x_{1}-\frac{h_{1}}{2}, x_{2}\right), \quad a_{2}(x)=k_{2}\left(x_{1}, x_{2}-\frac{h_{2}}{2}\right), \quad x=\left(x_{1}^{\left(i_{1}\right)}, x_{2}^{\left(i_{2}\right)}\right) \in \widehat{w}_{h} .
$$

This scheme has the local truncation error of $O\left(h^{2}\right), h^{2}=h_{1}^{2}+h_{2}^{2}, h_{p}=\max h_{p}^{\left(i_{p}\right)}, p=1,2$, if $u \in C^{4}\left(\Omega_{1} \cup \Omega_{2}\right)[10]$.

\subsection{Approximation of the Neumann transmission condition (2.2) on nonuniform} mesh. We begin by the consideration of the finite difference approximation of the transmission problem (2.1)-(2.3) with ideal contact conditions.

To approximate the Neumann interface condition (2.2), we use first the simplest finite difference equation

$$
a_{1}\left(h_{1}, x_{2}\right) y_{x_{1}}-a_{1}\left(0, x_{2}\right) y_{\bar{x}_{1}}=0, \quad x \in \Gamma_{\xi}^{h} \subset \widehat{w}_{h},
$$

where $\Gamma_{\xi}^{h}$ is the set of interface mesh points. Taking into account formulas (2.4) and (4.5), we get the following:

$$
a_{1}\left(h_{1}, x_{2}\right)=k_{1}\left(\frac{h_{1}}{2}, x_{2}\right), \quad a_{1}\left(0, x_{2}\right)=k_{1}\left(-\frac{h_{1}}{2}, x_{2}\right) .
$$


Let us now calculate the truncation error

$$
\psi_{h}:=a_{1}\left(h_{1}, x_{2}\right) u_{x_{1}}-a_{1}\left(0, x_{2}\right) u_{\bar{x}_{1}}
$$

of the finite difference approximation (4.6). For this aim, we use Taylor's formula on the right-hand side of (4.8):

$$
\begin{aligned}
\left.\psi_{h}\right|_{x_{1}=0}= & k_{1}^{(2)}\left(+0, x_{2}\right) \frac{\partial u}{\partial x_{1}}\left(+0, x_{2}\right)-k_{1}^{(1)}\left(-0, x_{2}\right) \frac{\partial u}{\partial x_{1}}\left(-0, x_{2}\right) \\
& +\left.\frac{h_{1}}{2} \frac{\partial}{\partial x_{1}}\left(k_{1}^{(2)}(x) \frac{\partial u}{\partial x_{1}}\right)\right|_{x_{1}=+0}+\left.\frac{h_{1}}{2} \frac{\partial}{\partial x_{1}}\left(k_{1}^{(1)}(x) \frac{\partial u}{\partial x_{1}}\right)\right|_{x_{1}=-0}+O\left(h_{1}^{2}\right) .
\end{aligned}
$$

Taking into account the Neumann interface condition (2.2), we can eliminate the first and the second terms on the right-hand side. Hence

$$
\left.\psi_{h}\right|_{x_{1}=0}=\frac{h_{1}}{2}\left[\left.\frac{\partial}{\partial x_{1}}\left(k_{1}^{(2)}(x) \frac{\partial u}{\partial x_{1}}\right)\right|_{x_{1}=+0}+\left.\frac{\partial}{\partial x_{1}}\left(k_{1}^{(1)}(x) \frac{\partial u}{\partial x_{1}}\right)\right|_{x_{1}=-0}\right]+O\left(h_{1}^{2}\right),
$$

and we get an $O(h)$ truncation error along the interface $\Gamma_{\xi}$.

Consider now the spectral case on an orthotropic material, when the heat conduction equation (2.1) has the form

$$
-\frac{\partial}{\partial x_{1}}\left(k_{1}(x) \frac{\partial u}{\partial x_{1}}\right)-\frac{\partial}{\partial x_{2}}\left(k_{2}(x) \frac{\partial u}{\partial x_{2}}\right)=F(x), \quad x \in \Omega
$$

where $k_{2}(x) \in C(\Omega)$ and $k_{1}(x)$ has the same form (2.4), that is, the heat conduction coefficient $k_{1}^{(i)}(x)$ is continuous, but the coefficient is discontinuous. In this case, by using (4.11) on the right-hand side of (4.10), we get

$$
\left.\psi_{h}\right|_{x_{1}=0}=-h_{1}\left[\frac{\partial}{\partial x_{2}}\left(k_{2}(x) \frac{\partial u}{\partial x_{2}}\right)+F\left(x_{1}, x_{2}\right)\right]_{x_{1}=0}+O\left(h_{1}^{2}\right)
$$

Taking into account the right-hand side of this expression, we consider the following approximation of the Neumann transmission condition:

$$
a_{1}\left(h_{1}, x_{2}\right) y_{x_{1}}-a_{1}\left(0, x_{2}\right) y_{\bar{x}_{1}}+h_{1}\left(a_{2} y_{\bar{x}_{2}}\right)_{\hat{x}_{2}}+h_{1} F(x)=0, \quad x \in \Gamma_{\xi}^{h}
$$

instead of (4.6). Here $a_{2}(x)=k_{2}\left(x_{1}, x_{2}-h_{2} / 2\right)$, according to (4.5).

This scheme has the local truncation error $O\left(h_{1}^{2}\right)$, as formula (4.12) shows. To analyse scheme (4.13), we divide both sides by $h_{1} \neq 0$ and use the above notations for finite derivatives. Then the finite difference equation has the following canonical form:

$$
-\left(a_{1} y_{\bar{x}_{1}}\right)_{\widehat{x}_{1}}-\left(a_{2} y_{\bar{x}_{2}}\right)_{\hat{x}_{2}}=F(x), \quad x \in \Gamma_{\xi}^{h} .
$$


This is identical to the scheme (4.4). Thus, due to the conservativeness of the finite difference schemes, even in the case of discontinuous coefficient $k_{1}(x)$, the scheme (4.14) has the same form (4.4) at the interface mesh points.

4.2. Approximation of the transmission conditions (3.10). Due to the discontinuity of the solution $u(x)$ across the interface, we introduce the following:

$$
y^{-}:=y^{-}\left(-0, x_{2}\right), \quad y^{+}:=y^{+}\left(+0, x_{2}\right) \quad\left(y^{-} \neq y^{+}\right)
$$

on the mesh points along the interface $\Gamma_{\xi}$. We approximate the interface conditions $(3.10)$ by the following finite difference equations:

$$
\sigma\left(y^{+}-y^{-}\right)=a_{1}\left(-0, x_{2}\right) y_{\bar{x}_{1}}, \quad \sigma\left(y^{+}-y^{-}\right)=a_{1}\left(h_{1}, x_{2}\right) y_{x_{1}}, \quad x \in \Gamma_{\xi}^{h} \subset \widehat{w}_{h},
$$

where the coefficients are defined by (4.5). To estimate the truncation error, we use the above Taylor formula:

$$
\psi_{h}:=\sigma[u]-a_{1}\left(0, x_{2}\right) u_{\bar{x}_{1}}=\sigma[u]-\left(k_{1}^{(1)} \frac{\partial u}{\partial x_{1}}\right)_{x_{1}=-0}+\frac{h_{1}}{2}\left[\frac{\partial}{\partial x_{1}}\left(k_{1}^{(1)} \frac{\partial u}{\partial x_{1}}\right)\right]_{x_{1}=-0}+O\left(h_{1}^{2}\right) .
$$

By the first Neumann transmission condition (3.10), the local truncation error on the mesh points of the interface is of $O\left(h_{1}\right)$. The same error has the second scheme (4.16).

In the case of the elliptic equation (4.11), we can use the above technique to construct the following schemes:

$$
\begin{gathered}
\sigma\left(y^{+}-y^{-}\right)-a_{1}\left(-0, x_{2}\right) y_{\bar{x}_{1}}+\frac{h_{1}}{2}\left(a_{2}\left(0, x_{2}\right) y_{\bar{x}_{2}}\right)_{\hat{x}_{2}}+\frac{h_{1}}{2} F\left(0, x_{2}\right)=0, \\
\sigma\left(y^{+}-y^{-}\right)-a_{1}\left(h_{1}, x_{2}\right) y_{x_{1}}-\frac{h_{1}}{2}\left(a_{2}\left(h_{1}, x_{2}\right) y_{x_{2}}\right)_{\hat{x}_{2}}-\frac{h_{1}}{2} F\left(h_{1}, x_{2}\right)=0, \quad x \in \Gamma_{\xi}^{h} .
\end{gathered}
$$

Both schemes (4.18) have a local truncation error of $O\left(h^{2}\right)$ on the mesh points of the interface $\Gamma_{\xi}$.

\section{Numerical examples}

The series of computational experiments are done to confirm the mathematical model of layered conductivities without isolation and with thin isolation with ideal contact conditions, as well as the expected accuracy of the presented finite difference approximations. We illustrate some results by the following two examples in which $k_{2}(x)=1$ is taken. 
Example 5.1. In this example, we study the verification of the finite difference scheme (4.4) approximating the elliptic problem (2.1)-(2.3) with discontinuous coefficient $k_{1}(x)$. For this problem, we use the following exact solution:

$$
u\left(x_{1}, x_{2}\right)= \begin{cases}\left(x_{1}^{2}+1\right) e^{x_{2}}, & \left(x_{1}, x_{2}\right) \in \Omega_{1} \\ \left(x_{1}^{2}+x_{1}+0.5\right) e^{x_{2}}, & \left(x_{1}, x_{2}\right) \in \Omega_{2},\end{cases}
$$

with appropriate Dirichlet condition, and the piecewise constant coefficient $k_{1}(x)$ is given by

$$
k_{1}(x)= \begin{cases}2, & \left(x_{1}, x_{2}\right) \in \Omega_{1} \\ 1, & \left(x_{1}, x_{2}\right) \in \Omega_{2}\end{cases}
$$

where $\Omega:=\left\{\left(x_{1}, x_{2}\right) \in \mathbb{R}^{2}: x_{1} \in(0,1), x_{2} \in(0,1)\right\}$ and $\xi=0.5$. For the function $u\left(x_{1}, x_{2}\right)$, $\left.u\left(x_{1}, 0.5\right)\right|_{x_{1}=\xi^{-}}=\left.u\left(x_{1}, 0.5\right)\right|_{x_{1}=\xi^{+}}=2.0609$ and

$$
\left[k_{1}^{(1)} \frac{\partial u\left(x_{1}, 0.5\right)}{\partial x_{1}}\right]_{x_{1}=\xi^{-}}=\left[k_{1}^{(2)} \frac{\partial u\left(x_{1}, 0.5\right)}{\partial x_{1}}\right]_{x_{1}=\xi^{+}}=3.297
$$

Numerical solution of the problem is obtained by using the transmission condition (1.5). The relative errors of the values $\left.u^{h}\left(x_{1}, 0.5\right)\right|_{x_{1}=\xi}=2.0773$ and $\left[k_{1} \partial u^{h}\left(x_{1}, 0.5\right) / \partial x_{1}\right]_{x_{1}=\xi}=$ 3.293 , obtained numerically, are found to be $0.8 \%$ and $0.2 \%$, respectively. The problem is solved on the uniform mesh $41 \times 41$ and the figures of the obtained solution and relative error are given in Figures 5.1(a) and 5.1(b).

Example 5.2. In this example, we study the verification of the asymptotic analysis and compare the solutions of problem (3.1)-(3.5). We use the following exact solution, for the transmission problem (3.1)-(3.5) in Figure 5.2(a):

$$
u\left(x_{1}, x_{2}\right)= \begin{cases}\left(-1.0499 x_{1}^{2}+x_{1}+1\right) e^{x_{2}}, & \left(x_{1}, x_{2}\right) \in \Omega_{1}, \\ \left(10.0364 x_{1}^{2}-4.266 x_{1}+1\right) e^{x_{2}}, & \left(x_{1}, x_{2}\right) \in \Omega_{\delta} \\ \left(-1.8988 x_{1}^{2}+2 x_{1}+1\right) e^{x_{2}}, & \left(x_{1}, x_{2}\right) \in \Omega_{2}\end{cases}
$$

with appropriate Dirichlet condition, and the discontinuous coefficient (3.9) is given by

$$
k_{1}(x)= \begin{cases}2, & \left(x_{1}, x_{2}\right) \in \Omega_{1} \\ 0.001, & \left(x_{1}, x_{2}\right) \in \Omega_{\delta} \\ 1, & \left(x_{1}, x_{2}\right) \in \Omega_{2}\end{cases}
$$

where $\Omega=\Omega_{1} \cup \Omega_{\delta} \cup \Omega_{2}$. The domains $\Omega_{1}, \Omega_{\delta}$, and $\Omega_{2}$ are taken as $\Omega_{1}:=\left\{\left(x_{1}, x_{2}\right) \in\right.$ $\left.\mathbb{R}^{2}: x_{1} \in(0,0.475), x_{2} \in(0,1)\right\}, \Omega_{\delta}:=\left\{\left(x_{1}, x_{2}\right) \in \mathbb{R}^{2}: x_{1} \in(0.475,0.525), x_{2} \in(0,1)\right\}$, 


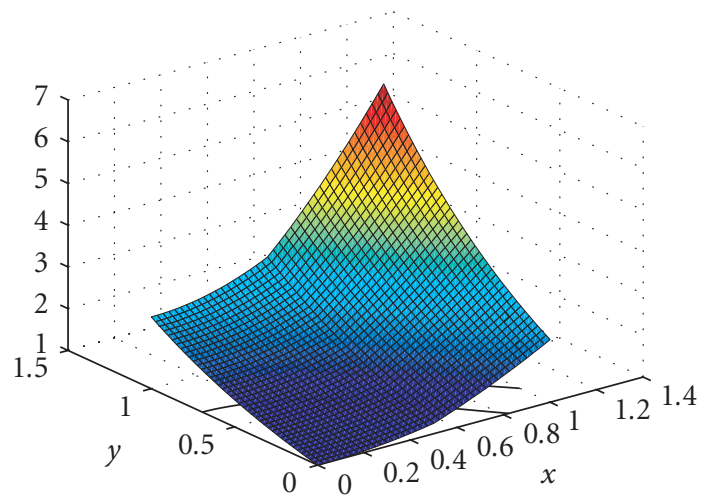

(a)

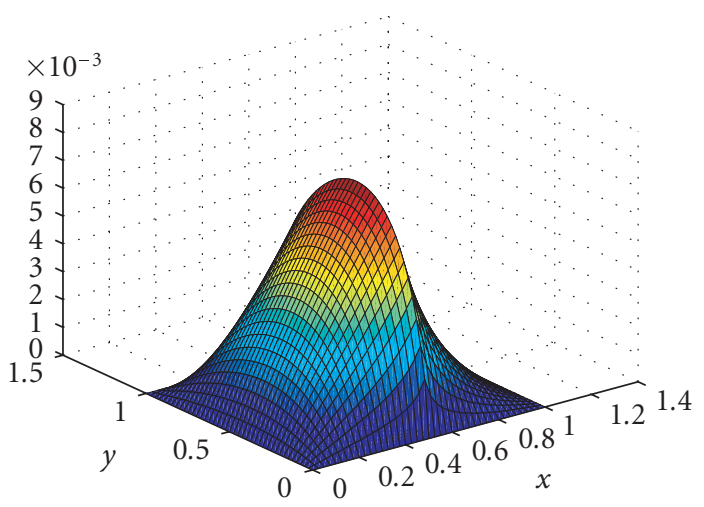

(b)

Figure 5.1. For the first example, the obtained solution and the relative error are given in part (a) and part (b), respectively.

and $\Omega_{2}:=\left\{\left(x_{1}, x_{2}\right) \in \mathbb{R}^{2}: x_{1} \in(0.525,1), x_{2} \in(0,1)\right\}$. In this case, $\Gamma_{\delta}^{-}:=\left\{\left(0.475, x_{2}\right)\right.$ : $\left.x_{2} \in(0,1)\right\}$ and $\Gamma_{\delta}^{+}:=\left\{\left(0.525, x_{2}\right): x_{2} \in(0,1)\right\}$. For the function $u\left(x_{1}, x_{2}\right)$, the evaluations $\left.u\left(x_{1}, x_{2}\right)\right|_{\left(x_{1}, x_{2}\right) \in \Gamma_{\delta}^{-}}=2.0413,\left.u\left(x_{1}, x_{2}\right)\right|_{\left(x_{1}, x_{2}\right) \in \Gamma_{\delta}^{+}}=2.517,\left[k_{1} \partial u\left(x_{1}, x_{2}\right) / \partial x_{1}\right]_{\left(x_{1}, x_{2}\right) \in \Gamma_{\delta}^{-}}=$ 0.008686 , and $\left[k_{1} \partial u\left(x_{1}, x_{2}\right) / \partial x_{1}\right]_{\left(x_{1}, x_{2}\right) \in \Gamma_{\delta}^{+}}=0.010345$ are obtained. The results of the numerical solution on different meshes are given in Table 5.1. The fourth and fifth columns of the table show the values of the fluxes on the interfaces $\Gamma_{\delta}^{-}$and $\Gamma_{\delta}^{+}$, respectively, for $\delta=0.025$. These results are in agreement with Proposition 3.1. Relative error of the obtained numerical solution is given in Figure 5.2(b). The figures of the cross sections of the approximate solution and relative error with the plane $x_{2}=0.5$ are given in Figures 5.3(a) and 5.3(b), respectively. The geometry of the behaviour of the relative error at the cross section $x_{2}=0.5$ for different thickness $\delta=h ; 2 h ; 3 h$ of the layer (isolation) is illustrated in Figure 5.4. The minimum relative error, in all cases, corresponds to the central point $x_{1}=0.5$ of the isolation, is as expected. 


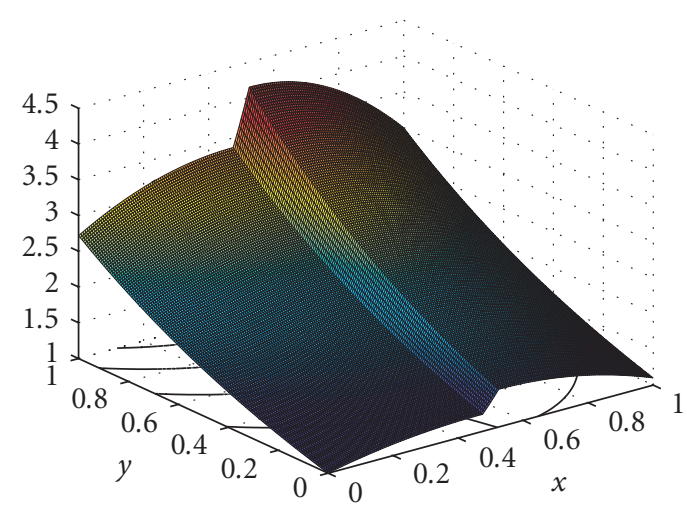

(a)

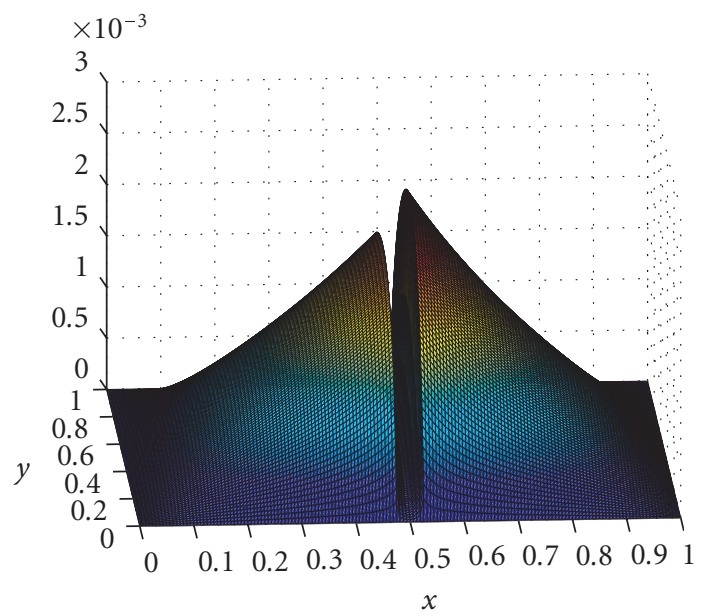

(b)

Figure 5.2. For the second example, the exact solution and the relative error of the obtained numerical solution are given in part (a) and part (b), respectively.

Table 5.1. Results of the numerical solution on different meshes.

\begin{tabular}{c|ccccc}
\hline Mesh & $u^{h}(0.475,0.5)$ & $u^{h}(0.525,0.5)$ & {$\left[k_{1} \frac{\partial u^{h}}{\partial x_{1}}\right]_{(0.475,0.5)}$} & {$\left[k_{1} \frac{\partial u^{h}}{\partial x_{1}}\right]_{(0.525,0.5)}$} & Max. Rel. Err. \\
\hline $41 \times 41$ & 2.0236 & 2.4903 & 0.00957 & 0.00910 & 0.0107 \\
$81 \times 81$ & 2.0326 & 2.5039 & 0.00932 & 0.00946 & 0.0053 \\
$161 \times 161$ & 2.0370 & 2.5105 & 0.00907 & 0.00981 & 0.0026 \\
\hline
\end{tabular}


16 Numerical analysis of steady state interface problems

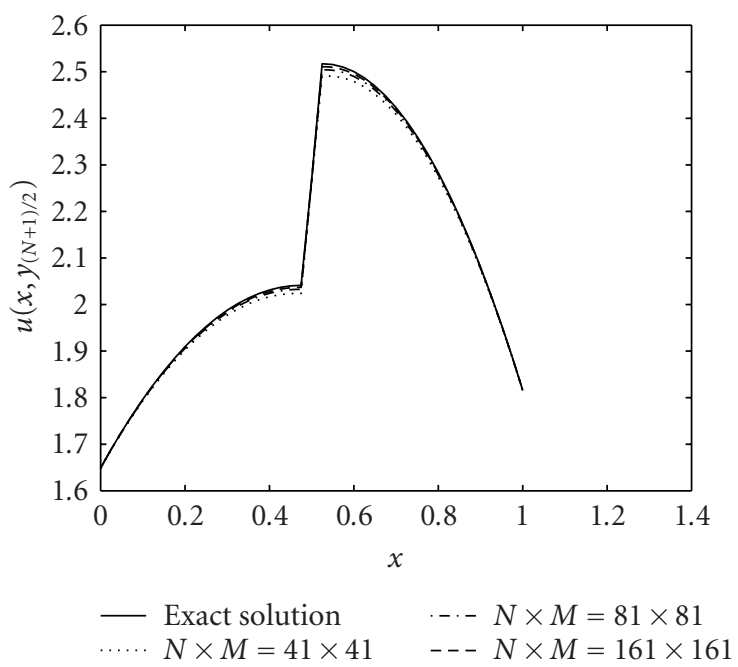

(a)

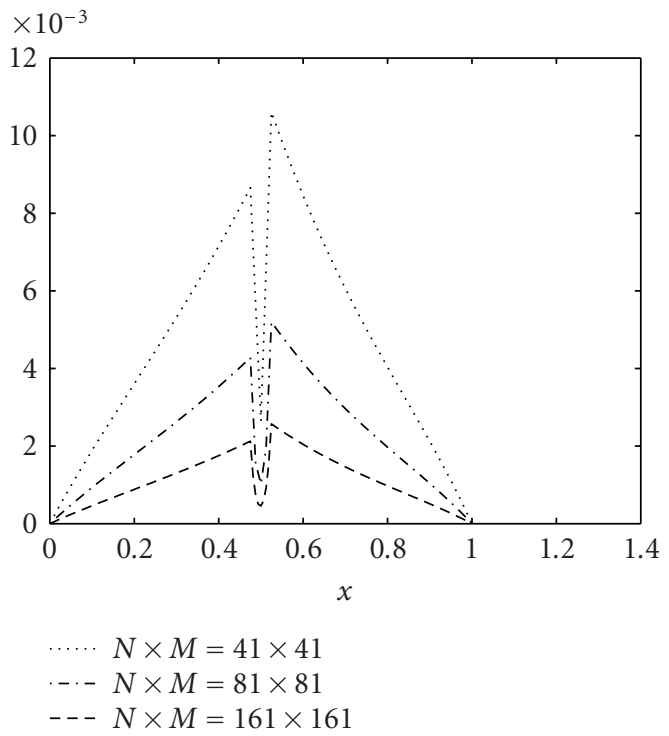

(b)

Figure 5.3. For the second example, the cross section of the approximate solution and the relative error with the plane $x_{2}=0.5$ are given in part (a) and part (b), respectively.

To have an overview of $p$, the order of convergence (experimentally) of each scheme, we chose two different step sizes $h_{1}$ and $h_{2}$. Then we calculated the corresponding errors, say $E_{1}$ and $E_{2}$, respectively. We assumed that $E_{1}=\operatorname{ch}_{1}^{p}$ and $E_{2}=\operatorname{ch}_{2}^{p}$. 


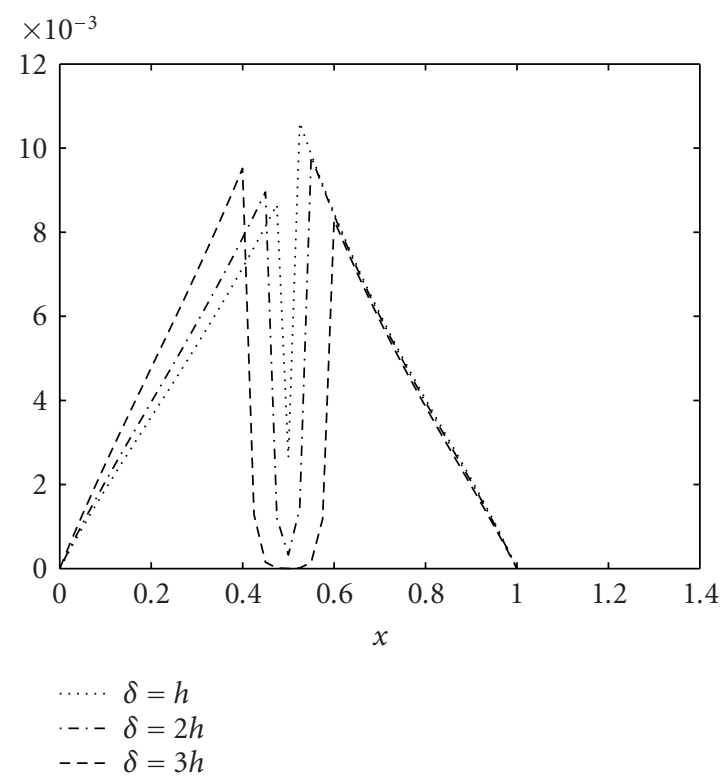

Figure 5.4. Geometric behaviour of the relative error at $x_{2}=0.5$.

Thus

$$
p=\frac{\ln \left(E_{1} / E_{2}\right)}{\ln \left(h_{1} / h_{2}\right)}
$$

The above formula was used to estimate the order of convergence for each finite difference method. The results were obtained via various values of $h$. We observed that the order of convergence in the domain $\Omega_{\delta}$ and $\Omega_{1} \cup \Omega_{2}$ were 1 and 2, respectively for Example 5.2.

\section{Acknowledgments}

The authors thank Professor Dr. Alemdar Hasanoglu (Hasanov) for the statement of the problem and helpful discussions. The authors also gratefully thank referees for reading the paper carefully and for their valuable suggestions that improved the manuscript.

\section{References}

[1] M. Costabel and M. Dauge, A singularly perturbed mixed boundary value problem, Communications in Partial Differential Equations 21 (1996), no. 11-12, 1919-1949.

[2] B. Engguist and J. C. Nedelec, Effective boundary conditions for electromagnetic scattering in thin layers, Rapport interne 278, Ecole Polytechnique-CMAP, Palaiseau, 1993.

[3] B. Engquist and A. Majda, Absorbing boundary conditions for the numerical simulation of waves, Mathematics of Computation 31 (1977), no. 139, 629-651.

[4] R. E. Ewing, Z. Li, T. Lin, and Y. Lin, The immersed finite volume element methods for the elliptic interface problems, Mathematics and Computers in Simulation 50 (1999), no. 1-4, 63-76. 
[5] A. I. Gasanov and I. E. Kaporin, Application of the elimination method in the solution of strongly elliptic systems by the finite element method, USSR Computational Mathematics and Mathematical Physics 26 (1986), no. 6, 837-850.

[6] D. Gilbarg and N. S. Trudinger, Elliptic Partial Differential Equations of Second Order, Springer, Berlin, 1977.

[7] P. Grisvard, Boundary Value Problems in Non-Smooth Domains, Pitman, London, 1985.

[8] T. Hagstrom and H. B. Keller, Exact boundary conditions at an artificial boundary for partial differential equations in cylinders, SIAM Journal on Mathematical Analysis 17 (1986), no. 2, 322341.

[9] R. J. LeVeque and Z. L. Li, The immersed interface method for elliptic equations with discontinuous coefficients and singular sources, SIAM Journal on Numerical Analysis 31 (1994), no. 4, 10191044.

[10] Z. Li, W.-C. Wang, I.-L. Chern, and M.-C. Lai, A new formulation for certain interface problems in polar coordinated and applications, to appear in SIAM Journal on Scientific Computing.

[11] A. A. Samarskii and V. B. Andreev, Difference Schemes for Elliptic Problems, Izdat. "Nauka", Moscow, 1976.

[12] A. N. Tikhonov and A. A. Samarskii, Homogeneous difference schemes, USSR Computational Mathematics and Mathematical Physics 1 (1962), no. 1, 5-67.

[13] E. Weinan, Selected Problems in Materials Science, Springer, New York, 2000.

[14] A. Wiegmann and K. P. Bube, The immersed interface method for nonlinear differential equations with discontinuous coefficients and singular sources, SIAM Journal on Numerical Analysis 35 (1998), no. 1, 177-200.

Z. Muradoglu Seyidmamedov: Applied Mathematical Sciences Research Center and Department of Mathematics, Kocaeli University, Umuttepe Campus, Izmit - Kocaeli 43800, Turkey

E-mail address: zahir@kou.edu.tr

Ebru Ozbilge: Department of Mathematics, Faculty of Science and Literature, Izmir University of Economics, 156 Sakarya Avenue, Balcova - Izmir 35330, Turkey

E-mail address: ebru.ozbilge@ieu.edu.tr 


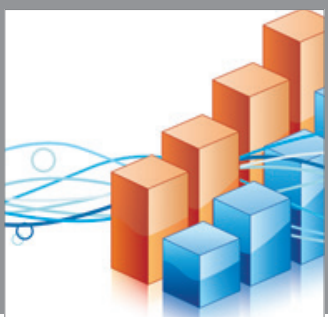

Advances in

Operations Research

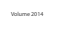

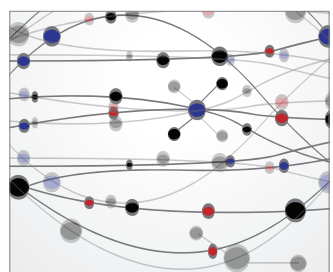

\section{The Scientific} World Journal
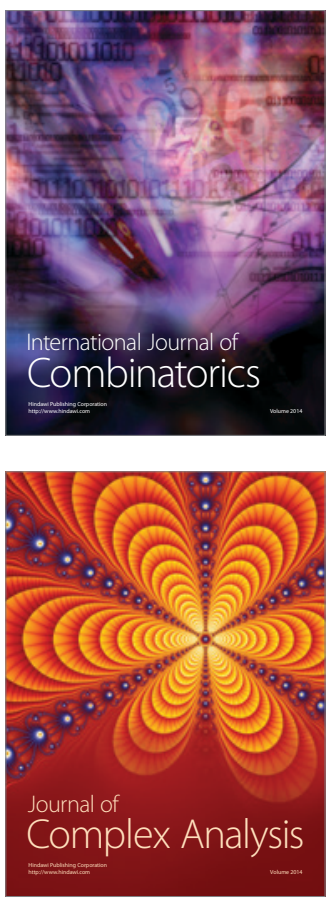

International Journal of

Mathematics and

Mathematical

Sciences
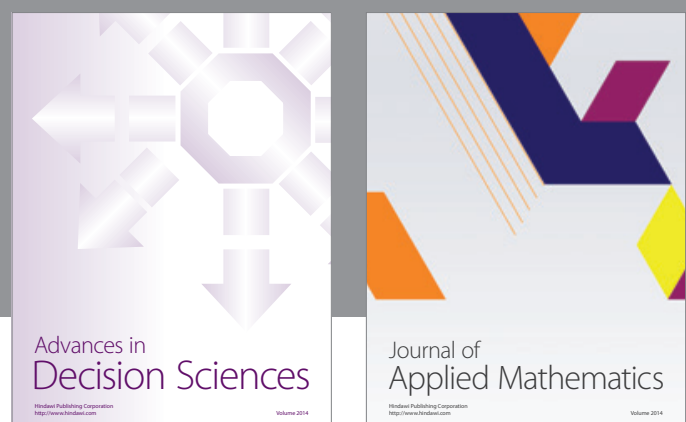

Journal of

Applied Mathematics
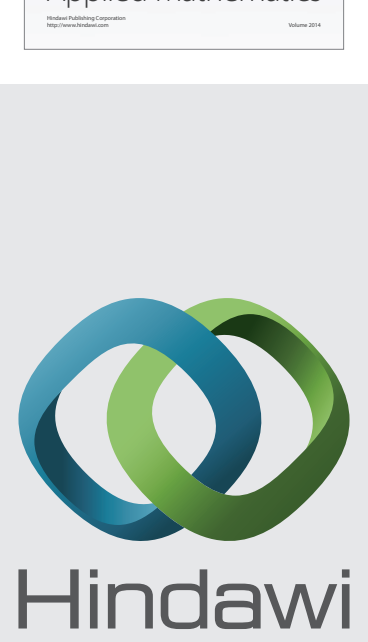

Submit your manuscripts at http://www.hindawi.com
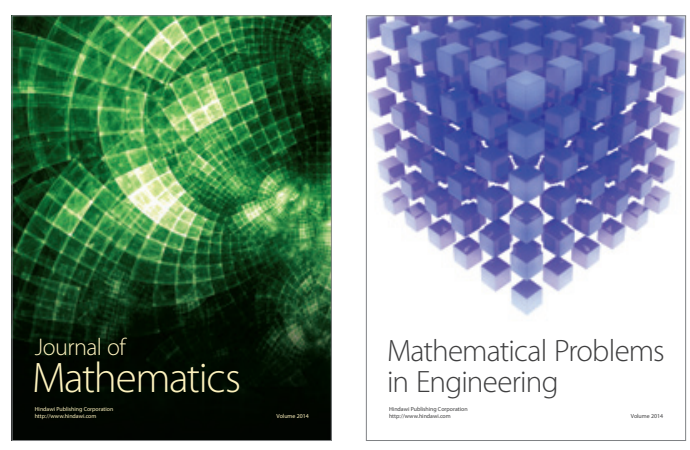

Mathematical Problems in Engineering
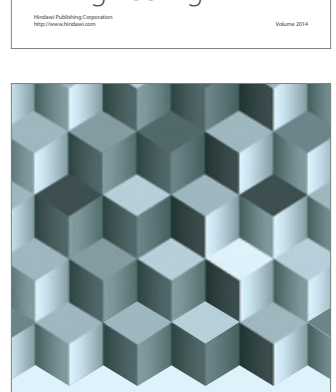

Journal of

Function Spaces
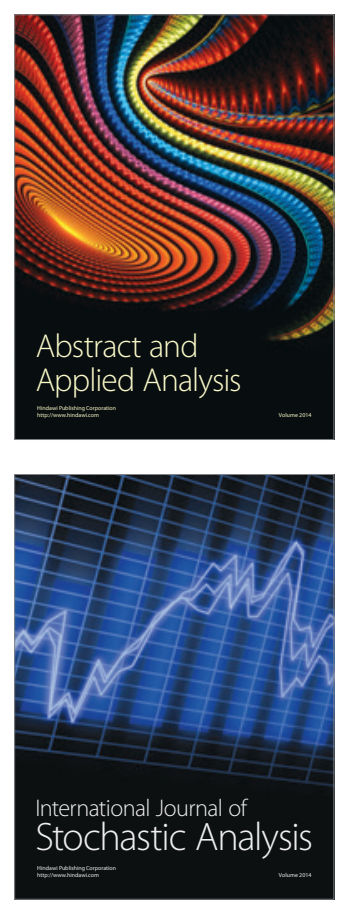

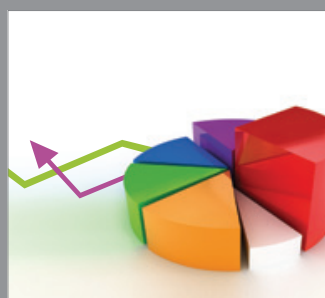

ournal of

Probability and Statistics

Promensencen
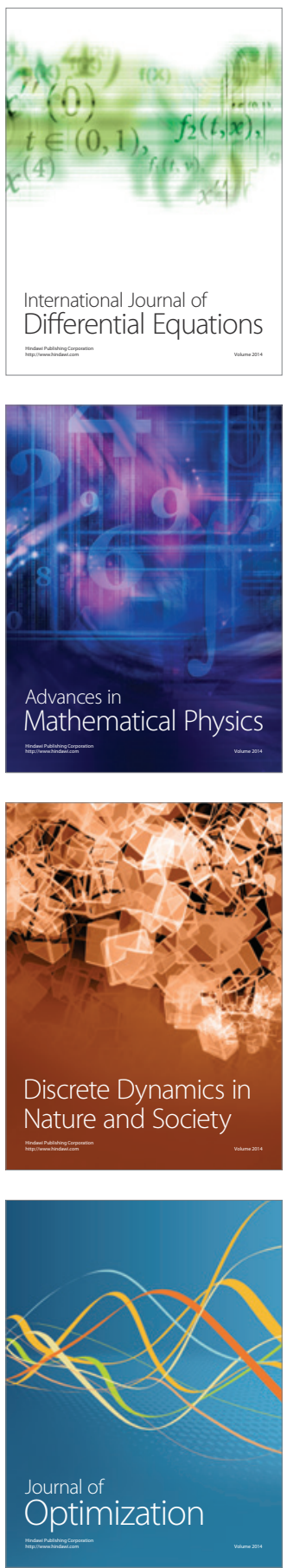